\title{
Perfil morfológico en levantadores de pesas federados de la región de Valparaíso, Chile
}

\author{
Morphological profile of federated weightlifters from the Valparaiso region, Chile \\ Héctor Fuentes-Barria ${ }^{*}$ orcid.org/0000-0003-0774-0848 \\ Sebastián Urbano-Cerda' ${ }^{1}$ orcid.org/0000-0003-0508-6985 \\ Raúl Aguilera-Eguía² orcid.org/0000-0002-4123-4255 \\ Catalina González-Wong ${ }^{3}$ orcid.org/0000-0003-0360-8567
}

Valentina Vera-Aguirre ${ }^{4}$ orcid.org/0000-0003-3050-5192

\begin{abstract}
1. Escuela de Ciencias del Deporte y Actividad Física, Facultad de Salud. Universidad Santo Tomás. Santiago, Chile.
2. Departamento de Salud Pública, Facultad de Medicina, Carrera de kinesiología. Universidad Católica de la Santísima Concepción. Concepción, Chile

3. Enfermería, Facultad de Medicina, Universidad del Desarrollo. Santiago, Chile.

4. Escuela de Nutrición y Dietética, Facultad de Ciencias para el Cuidado de la Salud. Universidad San Sebastián. Santiago, Chile.
\end{abstract} federados de la región de Valparaíso, Chile. Univ. Salud. 2021;23(2):162-167. DOI: https://doi.org/10.22267/rus.212302.228

\section{Resumen}

Introducción: En la halterofilia los perfiles antropométricos permiten una mejor planificación y ejecución del entrenamiento deportivo. Objetivo: Describir y comparar según sexo, el perfil antropométrico y somatotipo de levantadores de pesas federados de la región de Valparaíso, Chile. Materiales y métodos: Estudio descriptivo, transversal. Participaron 40 adultos levantadores de pesas. La evaluación antropométrica fue realizada utilizando el protocolo de medición y marcaje de la Sociedad Internacional de Avances en Cineantropometría, método pentacompartimental de Ross y Kerr y somatotipo de Heath-Carter. Se aplicó la T de Student para muestras independientes y U Mann-Whitney para comparar características antropométricas entre grupos. El poder estadístico y el tamaño efecto se calculó con "d" de Cohen. Resultados: Existen diferencias significativas entre grupos, para masa muscular relativa $(p=0,003 ; \mathrm{d}=0,96)$ y absoluta $(p \leq 0,001 ; \mathrm{d}=2,42)$; masa adiposa relativa $(p \leq 0,001 ; \mathrm{d}=1,46)$; masa ósea absoluta $(p \leq 0,001 ; \mathrm{d}=1,41)$; masa piel relativa $(p \leq 0,001 ; \mathrm{d}=1,96)$ y absoluta $(p \leq 0,001 ; \mathrm{d}=0,97)$; masa residual relativa $(p=0,006 ; \mathrm{d}=1,08)$ y absoluta $(p \leq 0,001 ; \mathrm{d}=2,09)$, mientras que la distribución del somatotipo clasifica al grupo masculino como Endo-Mesomorfo y al femenino como Mesomorfo-Endomorfo, observando diferencias significativas en el mesomorfismo ( $p \leq 0,001$; $\mathrm{d}=1,48)$. Conclusiones: Existe un predominio del somatotipo mesomórfico, existiendo diferencias significativas en los componentes de composición corporal.

Palabras clave: Levantamiento de peso; antropometría; somatotipos; rendimiento atlético. (Fuente: DeCS, Bireme).

\begin{abstract}
Introduction: Anthropometric profiles allow better planning and execution of sports training in weightlifting. Objective: To describe and compare, according to gender, the anthropometric profile and somatotype of federated weightlifters from the Valparaiso region of Chile. Materials and methods: A descriptive cross-sectional study that included 40 adult weightlifters. The anthropometric assessment was carried out using the measurement and marking protocol of the International Society for Advances in Kinanthropometry, Ross and Kerr pentacompartmental method, and Heath-Carter somatotype. A Student's t test was applied for independent samples and U Mann-Whitney was used to compare anthropometric characteristics between groups. The statistical power and effect size were calculated using Cohen's "d". Results: There are significant differences between groups for: relative $(p=0.003 ; \mathrm{d}=0.96)$ and absolute $(p \leq 0.001 ; \mathrm{d}=2.42)$ muscle mass; relative fat mass $(p \leq 0.001 ; \mathrm{d}=1.46)$; absolute bone mass $(p \leq 0.001 ; \mathrm{d}=1.41)$; relative $(p \leq 0.001 ; \mathrm{d}=1.96)$ and absolute $(p \leq 0.001 ; \mathrm{d}=0.97)$ skin mass; relative $(p=0.006 ; \mathrm{d}=1.08)$ and absolute $(p \leq 0.001 ; \mathrm{d}=2.09)$ residual mass. Based on the somatotype distribution, the male and female groups were classified as EndoMesomorph and Mesomorph-Endomorph, respectively, which showed significant differences in mesomorphism $(p \leq 0.001$; $\mathrm{d}=1.48)$. Conclusions: There is a predominance of the mesomorphic somatotype, with significant differences in the components of the body composition.
\end{abstract}

Keywords: Weight lifting; anthropometry; somatotypes; athletic performance. (Fuente: DeCS, Bireme).

\footnotetext{
*Autor de correspondencia 


\section{Introducción}

La halterofilia es un deporte olímpico cuyo objetivo es lograr levantar una barra con el máximo peso posible desde el suelo hasta por encima de la cabeza, mediante un movimiento de triple extensión (rodilla, cadera, brazos)(1). Esta consta de dos tipos de levantamientos, el arranque y el envión; en el arranque la barra se eleva directamente desde el suelo hasta encima de la cabeza con un solo movimiento, mientras que en el envión se requiere de dos movimientos (clean y jerk), en los cuales en primera instancia se eleva la barra desde el suelo hasta los hombros, para posteriormente flexionar un poco las piernas y empujar la barra sobre la cabeza con una total extensión de los brazos ${ }^{(2)}$.

En la actualidad la Federación Chilena de Levantamiento de Pesas (FECHIPE) conforma las selecciones sub-15, sub-17, sub-23, juvenil y adulta, siguiendo la normativa impuesta por la Federación Internacional de Levantamiento de Pesas (IWF por su sigla en inglés) que subdivide a los deportistas según su peso corporal en categoría masculina $(55$, $61,67,73,81,89,96,102,109$ у >109 kg) y femenina $(45,49,55,59,64,71,76,81,87 \mathrm{y}>87 \mathrm{~kg})$ caracterizándose ambas por requerir un elevado componente físico que genera la adquisición de adaptaciones propias al entrenamiento durante el periodo de especialización deportiva, además de algunas características antropométricas condicionantes del rendimiento deportivo como el somatotipo(3-6). En este sentido, el somatotipo representa un método cuantitativo estandarizado que evalúa las dimensiones corporales y características físicas en poblaciones atléticas y no atléticas, constituyendo un elemento relevante al momento de planificar estrategias nutricionales o programas de entrenamiento individualizados, que busquen generar cambios morfológicos y mejoras del rendimiento deportivo relacionadas a condiciones morfológicas favorables para la práctica de ciertos deportes (7-11).

A nivel mundial existen algunos estudios que proporcionan valores referenciales asociados al somatotipo y mejoras al rendimiento físico en halterofilia(2,10-13), no obstante, en Chile existen escasos antecedentes sobre las características antropométricas para esta población(14).

La importancia de la composición corporal en halterofilia radica en la necesidad de lograr levantar el máximo peso posible manteniendo una baja masa grasa y una elevada masa magra, puesto que este deporte al estar normado según categorías de peso corporal limita el aumento excesivo de la masa corporal total(1-3). En este contexto, la masa grasa y magra constituyen aspectos fundamentales que deben controlarse regularmente durante el desarrollo atlético, debido a que influyen el desarrollo de la fuerza y potencia muscular en todas las edades y categorías de peso, por cuanto la identificación de estas características podría proporcionar una ventaja en la optimización y planificación del entrenamiento deportivo, así como en las decisiones de los deportistas y entrenadores ${ }^{(2-}$ 4).

Por esta razón, esta investigación se planteó como objetivo describir y comparar según sexo el perfil antropométrico y somatotipo de levantadores de pesas federados de la región de Valparaíso, Chile.

\section{Materiales y métodos}

Estudio tipo descriptivo, de diseño observacional y de corte transversal, desarrollado en las dependencias del gimnasio Kraken FC ubicado en las comunas de Quillota y Villa Alemana (Región de Valparaíso, Chile). Los participantes de este estudio fueron 40 adultos (27 hombres y 13 mujeres) afiliados a la Federación Chilena de Levantamiento de Pesas, quienes fueron seleccionados de manera no probabilística por conveniencia según los siguientes criterios de inclusión:

- Adultos entre 18 y 65 años.

- Estar entre las categorías de peso IWF 56 a 96 kg para hombres y 45 a $75 \mathrm{~kg}$ para mujeres.

- No presentar prótesis o implantes metálicos.

- Presentar una ingesta de agua o alimentos $>2$ horas previa a la evaluación antropométrica.

- Presentar una ingesta de alcohol o café >8 horas previa evaluación antropométrica.

- Presentar actividad física intensa >24 horas previa evaluación antropométrica.

- Presentar un nivel de entrenamiento >3 días/semana.

- No presentar síndrome premenstrual, posibilidad de embarazo y lactancia.

- No presentar imposibilidad de vaciar la vejiga previa evaluación antropométrica. 
- No presentar ingesta de medicamentos de tipo diuréticos o corticoides que pudieran alterar la composición corporal.

- Aceptar participar voluntariamente del estudio y firmar un consentimiento informado que autoriza el uso de la información con fines de investigación científica hasta por cinco años.

\section{Procedimiento e instrumentos}

Para las mediciones se utilizó el kit antropométrico marca Cescorf validado por la Sociedad Internacional de Avances en Cineantropometría (ISAK, por su sigla en inglés), compuesto por una balanza digital Seca con precisión de 100 gr, estadiómetro portátil Seca de $220 \mathrm{~cm}$, cinta métrica con precisión de $1 \mathrm{~mm}$, segmómetro con precisión de $1 \mathrm{~mm}$ y un plicómetro con precisión de $1 \mathrm{~mm}$.

Los sujetos fueron evaluados bajo condiciones normales de temperatura y humedad por un profesional capacitado en ISAK nivel II, quien realizó el marcaje y medición del perfil completo de todos los participantes. Posteriormente, los datos fueron procesados en una planilla Excel según la posición anatómica, considerando un $1 \%$ de error en la técnica de medición para las variables basales peso corporal $(\mathrm{kg})$, talla sentado $(\mathrm{cm})$, talla parado $(\mathrm{cm})$ e índice de masa corporal $\left(\mathrm{kg} / \mathrm{m}^{2}\right)$ y un $5 \%$ para la sumatoria de seis pliegues cutáneos (tricipital, subescapular, supraespinal, abdominal, muslo y pierna)(15).

La composición corporal se evaluó mediante el método pentacompartimental de Ross y Kerr que fracciona el peso corporal total en cinco masas (muscular, adiposa, ósea, piel y residual), comparando los resultados con los valores $\mathrm{Z}$ del modelo Phantom de Ross \& Marfell-Jones, mientras que el somatotipo de Heath-Carter que fracciona el cuerpo en tres componentes (endomorfo, mesomorfo y ectomorfo) se determinó utilizando las mediciones antropométricas del perfil completo ISAK considerando para su clasificación el grado de manifestación de cada componente(14-16).

\section{Análisis estadístico}

Los datos fueron analizados con el software estadístico SPSS versión 23.0 en conjunto con el software G*Power versión 3.1 para sistema operativo Windows. Se calculó la media (X), mediana (Me), desviación estándar (DE) y rango intercuartil (RIQ) para describir las variables. La distribución y homocedasticidad de los datos fueron determinadas con las pruebas de Shapiro-Wilk y Levene respectivamente. Se aplicó la prueba $T$ de Student para muestras independientes para comparar las variables paramétricas (masa muscular, masa adiposa, masa piel, masa residual, endomorfismo y mesomorfismo) y la prueba U Mann Whitney para las variables no paramétricas (masa ósea y ectomorfismo). El tamaño del efecto se determinó con la " $d$ " de cohen considerando un efecto pequeño $(0,2)$, moderado $(0,5)$ y grande $(0,8)$, mientras que la potencia estadística se evaluó con $1-\beta$ considerando un nivel mínimo de 0,80 . Finalmente, para todos los análisis se consideró un nivel alfa 0,05.

\section{Consideraciones éticas}

Esta investigación fue aprobada por el Comité de Ética de la Universidad Santo Tomás, Chile (folio 128-19), quien revisó que los procedimientos de la investigación siguieran las consideraciones éticas de la declaración de Helsinki del año 2013.

\section{Resultados}

La Tabla 1 presenta los valores para las variables básicas de edad, masa corporal, talla de pie, talla sentado, índice de masa corporal y sumatoria de seis pliegues cutáneos de acuerdo con el sexo.

Tabla 1. Valores basales en levantadores de pesas de la región de Valparaíso, Chile

\begin{tabular}{|c|c|c|c|c|c|c|c|c|}
\hline \multirow{2}{*}{ Variables } & \multicolumn{4}{|c|}{ Grupo masculino $(n=27)$} & \multicolumn{4}{|c|}{ Grupo femenino (n=13) } \\
\hline & $\mathbf{X}$ & DE & Me & RIQ & $\mathbf{X}$ & DE & Me & RIQ \\
\hline Edad (años) & 29 & 6,8 & 28,4 & 7,1 & 28 & 2,5 & 27 & 4,3 \\
\hline Masa corporal (kg) & 75,8 & 9,2 & 76 & 16,8 & 57,5 & 8 & 56 & 12 \\
\hline Talla de pie $(\mathrm{cm})$ & 170,5 & 5,1 & 170,7 & 5,9 & 158,5 & 5,5 & 160 & 8,5 \\
\hline Talla sentado $(\mathrm{cm})$ & 89,3 & 2,9 & 90,1 & 3,4 & 84,6 & 3,1 & 85,4 & 5,8 \\
\hline Índice de masa corporal $\left(\mathrm{kg} / \mathrm{m}^{2}\right)$ & 26,1 & 3,1 & 26,2 & 5,4 & 22,9 & 2,8 & 22,1 & 3,9 \\
\hline Sumatoria 6 pliegues cutáneos (mm) & 80,6 & 30,6 & 90 & 39,5 & 87,9 & 21,8 & 83 & 26,5 \\
\hline
\end{tabular}

n: número muestral; X: media; DE: desviación estándar; Me: mediana; RIQ: rango intercuartil. 
Al comparar los valores de los cinco componentes del método pentacompartimental se observa que existen diferencias significativas y un tamaño de efecto grande entre grupos para, masa muscular relativa $(p=0,003 ; d=0,96)$ y absoluta $(p \leq 0,001 ; d=$ $2,42)$; masa adiposa relativa $(p \leq 0,001 ; d=1,46)$; masa ósea absoluta $(p \leq 0,001 ; d=1,41)$; masa piel relativa $(p \leq 0,001 ; d=1,96)$ y absoluta $(p \leq 0,001 ; d=0,97)$; masa residual relativa $(p=0,006 ; d=1,08)$ y absoluta $(p \leq 0,001 ; d=2,09)$. En cuanto a la distribución del somatotipo solo se observan diferencias significativas y un tamaño de efecto grande en el componente mesomórfico $(p<0,001 ; d=1,48)$, además de una clasificación Endo-Mesomorfo para el grupo masculino y Mesomorfo-Endomorfo para el femenino (Tabla 2).

Tabla 2.Componentes de masa corporal y somatotipo según sexo

\begin{tabular}{|c|c|c|c|c|c|c|c|c|c|c|c|c|}
\hline \multirow{2}{*}{\multicolumn{2}{|c|}{ Componentes }} & \multicolumn{4}{|c|}{ Grupo masculino $(n=27)$} & \multicolumn{4}{|c|}{ Grupo femenino $(n=13)$} & \multicolumn{3}{|c|}{ Estadísticos } \\
\hline & & \multirow{2}{*}{$\frac{\mathbf{X}}{50,2}$} & \multirow{2}{*}{$\frac{\mathrm{DE}}{5,6}$} & \multirow{2}{*}{$\begin{array}{l}\text { Me } \\
50,9\end{array}$} & \multirow{2}{*}{$\begin{array}{r}\text { RIQ } \\
9,6\end{array}$} & \multirow{2}{*}{$\frac{X}{46}$} & \multirow{2}{*}{$\frac{\text { DE }}{2,7}$} & \multirow{2}{*}{$\frac{\text { Me }}{45,9}$} & \multirow{2}{*}{$\frac{\text { RIQ }}{3,8}$} & \multirow{2}{*}{$\begin{array}{r}\text { Valor } \boldsymbol{p} \\
0,003^{*}\end{array}$} & \multirow{2}{*}{$\frac{\boldsymbol{1}-\boldsymbol{\beta}}{0,79}$} & \multirow{2}{*}{$\begin{array}{l}\begin{array}{l}\text { Tamaño de } \\
\text { efecto }\end{array} \\
0,96\end{array}$} \\
\hline Muscular & $\%$ & & & & & & & & & & & \\
\hline & Kg & 38,1 & 6,5 & 37,3 & 9,5 & 25,3 & 3,7 & 25,1 & 3,6 & $\leq 0,001^{*}$ & 0,99 & 2,42 \\
\hline \multirow[t]{2}{*}{ Adiposa } & $\%$ & 24 & 4,8 & 24,7 & 5,6 & 31,1 & 4,9 & 29,4 & 5,2 & $\leq 0,001^{*}$ & 0,99 & 1,46 \\
\hline & Kg & 18,3 & 4,3 & 19 & 7,6 & 17,2 & 3,7 & 16 & 4,8 & 0,442 & 0,12 & 0,27 \\
\hline \multirow[t]{2}{*}{ Ósea } & $\%$ & 9,3 & 2,8 & 9 & 1,8 & 8,5 & 2,1 & 8,7 & 1,6 & 0,544 & 0,15 & 0,32 \\
\hline & Kg & 7 & 1,8 & 6,6 & 1,5 & 4,9 & 1,1 & 4,9 & 1,3 & $\leq 0,001^{*}$ & 0,98 & 1,41 \\
\hline \multirow[t]{2}{*}{ Piel } & $\%$ & 4,8 & 0,4 & 4,8 & 0,5 & 5,8 & 0,6 & 5,8 & 0,9 & $\leq 0,001^{*}$ & 0,99 & 1,96 \\
\hline & Kg & 3,6 & 0,3 & 3,7 & 0,4 & 3,2 & 0,5 & 3,1 & 0,6 & $\leq 0,001^{*}$ & 0,80 & 0,97 \\
\hline \multirow[t]{2}{*}{ Residual } & $\%$ & 11,5 & 1,5 & 11,2 & 1,7 & 9,4 & 2,3 & 10,1 & 3,5 & $0,006^{*}$ & 0,88 & 1,08 \\
\hline & Kg & 8,8 & 2 & 8,8 & 2 & 5,2 & 1,4 & 5,7 & 2,1 & $\leq 0,001^{*}$ & 0,99 & 2,09 \\
\hline \multicolumn{2}{|c|}{ Endomorfo } & 3,9 & 1,4 & 4 & 2,2 & 4,3 & 1,1 & 4,2 & 1,3 & 0,296 & 0,15 & 0,32 \\
\hline \multicolumn{2}{|c|}{ Mesomorfo } & 6,2 & 1,2 & 6,1 & 1,9 & 4,5 & 1,1 & 4,4 & 1,4 & $\leq 0,001^{*}$ & 0,99 & 1,48 \\
\hline \multicolumn{2}{|c|}{ Ectomorfo } & 1,3 & 1 & 1 & 1,7 & 1,7 & 1 & 1,9 & 1,7 & 0,174 & 0,20 & 0,40 \\
\hline \multicolumn{2}{|c|}{ Clasificación } & \multicolumn{4}{|c|}{ Endo-Mesomorfo } & \multicolumn{4}{|c|}{ Mesomorfo-Endomorfo } & & & \\
\hline
\end{tabular}

n: número muestral; X: media; DE: desviación estándar; Me: mediana; RIQ: rango intercuartil; *: diferencia significativa bilateral considerando p<0,05; 1$\beta$ : poder estadístico.

\section{Discusión}

Los hallazgos estadísticamente significativos en los cinco componentes de la masa corporal: muscular ( $p=0,003$ relativa; $p \leq 0,001$ absoluta), adiposo ( $p \leq 0,001$ relativa), óseo ( $p \leq 0,001$ absoluta), piel ( $p \leq 0,001$ relativa; $p=0,001$ absoluta) y residual ( $p \leq 0,006$ relativa; $p \leq 0,001$ absoluta), además del marcado componente mesomórfico $(p \leq 0,001)$ pueden explicarse debido a que el gasto energético asociado al nivel de entrenamiento puede determinar la composición corporal(2,17).

En este contexto, los 5 componentes de la composición corporal de William D. Ross y Deborah Kerr, corroboran que el sexo masculino posee una mayor masa muscular que le permite desarrollar mayores niveles de fuerza, mientras que el sexo femenino posee una facilidad para acumular tejido adiposo producto de diferentes factores biológicos(18,19). Estas diferencias son mediadas por el desarrollo musculoesquelético y pueden comprobarse al observar los mayores valores basales en las variables talla sentado, talla parado, masa corporal e Índice de masa corporal para el grupo masculino o la mayor sumatoria de seis pliegues cutáneos para el caso del grupo femenino. Las variaciones sobre la masa de piel pueden ser justificadas debido a que el sexo masculino posee un colágeno mucho más compacto producto de la acción moduladora de las hormonas sexuales lo que implica 
mayores cambios absolutos(20). Respecto a esto, las hormonas sexuales también pueden explicar en parte las diferencias no significativas sobre la masa ósea relativa y significativas para la masa ósea absoluta, puesto que la densidad mineral ósea y la distribución de la masa corporal dependen de hormonas como la testosterona la cual puede regular cambios ante una masa absoluta equivalente(21). Por otro lado, las diferencias en torno a la masa residual podrían explicarse por la capacidad de retención de líquidos internos atribuidos a los controles de pesaje característicos de este tipo de deportes(22).

En cuanto al somatotipo, los hallazgos de este y otros estudios plantean que el desarrollo del tejido adiposo representa una desventaja para el rendimiento óptimo deportivo, puesto que un bajo componente mesomórfico supone una facilidad para aumentar el desarrollo del tejido adiposo por causa de la dieta energética elevada, a modo de compensar la falta de desarrollo muscular en levantadores con un nivel de entrenamiento normal o bajo(2,11-14). En este sentido, los antecedentes antropométricos teóricos propuestos por Heath y Carter están determinados por múltiples factores genéticos y ambientales, no obstante, se ha aceptado que el componente mesomorfo se puede relacionar directamente con el desarrollo de la masa y fuerza muscular, mientras que la baja linealidad (ectomorfismo) se puede asociar inversamente al desarrollo del endomorfismo y mesomorfismo $(2,17)$.

Todas las diferencias observadas en los resultados antropométricos entre los sexos masculino y femenino, cobran una gran implicancia en levantadores de pesas, debido a que las masas adiposa y magra determinan las características propias del somatotipo que diferencian a los atletas de élite de aquellos que no lo son(2,10,13).

En este contexto, la evidencia científica ha planteado que una alimentación incorrecta podría llegar a condicionar la composición corporal y el rendimiento deportivo en levantadores de pesas, motivo por el cual las recomendaciones tradicionales para mantener un balance energético han propuesto un consumo de entre 4 a $7 \mathrm{~g} / \mathrm{kg}$ de peso de carbohidratos y 1,6 a $2 \mathrm{~g} / \mathrm{kg}$ de peso de proteínas como una alternativa para cubrir los requerimientos de este deporte, mientras que las recomendaciones actuales se han centrado sobre el postentrenamiento, sugiriendo el uso de una combinación de entre 0,8 a $1,2 \mathrm{~g} / \mathrm{kg}$ de peso de carbohidratos con $0,4 \mathrm{~g} / \mathrm{kg}$ de peso de proteínas para procurar disminuir la masa grasa, aumentar el tejido magro y por consecuencia mejorar el rendimiento deportivo disminuyendo las diferencias antropométricas reportadas en el presente estudio(2).

\section{Conclusiones}

Existen diferencias estadísticamente significativas en las cinco variables de composición corporal excepto la masa adiposa absoluta y ósea relativa, así como un somatotipo mesomórfico predominante. En este contexto, las intervenciones actuales proponen centrarse en disminuir la masa grasa, aumentar el tejido magro en ambos sexos con el objetivo de mejorar el rendimiento deportivo disminuyendo las diferencias antropométricas; sin embargo, la escasez de investigaciones similares en población chilena no permite establecer un perfil morfológico ni estrategias específicas orientadas a optimizar los niveles de fuerza e hipertrofia relacionados al rendimiento deportivo en levantadores de pesas.

\section{Limitaciones del estudio}

Esta investigación se encuentra limitada por la heterogeneidad del tamaño muestral (27 hombres y 13 mujeres), además de la baja potencia estadística que aumentó la probabilidad de presentar un error tipo II en 4 de las 13 variables analizadas. No obstante, a pesar de estas limitaciones la significancia estadística obtenida y el gran tamaño de efecto en las 9 variables restantes reflejan las diferencias observadas en la composición corporal de la muestra estudiada.

Agradecimientos: A todos quienes participaron en este estudio.

Conflicto de intereses: Los autores declaramos que no tener ningún tipo de conflicto de interés.

\section{Referencias}

1. Suchomel TJ, Comfort P, Stone MH. Weightlifting pulling derivatives: Rationale for Implementation and Application. Sports Med. 2015;45(6):823-39. doi: 10.1007/s40279-0150314-y.

2. Martínez-Rodríguez A, Tundidor-Duque RM, Alcaraz PE, Rubio-Arias JA. Estrategias dietéticas y composición corporal en halterofilia de élite: Revisión sistemática. Rev. Esp Nutr Hum Diet. 2017;21(3):237-47. doi: 10.14306/renhyd.21.3.353.

3. Participants. International Weightlifting Federation [Internet]. [cited 2020 Aug 31]. Disponible en: https://www.iwf.net/weightlifting_/participants/ 
4. Otto WH, Coburn JW, Brown LE, Spiering BA. Effects of weightlifting vs. kettlebell training on vertical jump, strength, and body composition. J Strength Cond Res. 2012;26(5):1199-202.

10.1519/JSC.0b013e31824f233e.

5. Crewther BT, Obmiński Z, Cook CJ. Serum cortisol as a moderator of the relationship between serum testosterone and olympic weightlifting performance in real and simulated competitions. Biol Sport. 2018;35(3):215-21. doi:10.5114/biolsport.2018.74632.

6. Storey A, Smith HK. Unique aspects of competitive weightlifting. Sport Med. 2012;42(9):769-90. doi: $10.1007 /$ bf03262294.

7. Ryan-Stewart H, Faulkner J, Jobson S. The influence of somatotype on anaerobic performance. Barbosa TM, editor. PLoS One. 2018;13(5):e0197761. doi: 10.1371/journal.pone.0197761.

8. Fields JB, Metoyer CJ, Casey JC, Esco MR, Jagim AR, Jones MT. Comparison of body composition variables across a large sample of national collegiate athletic association women athletes from 6 competitive sports. J Strength Cond Res. 2018;32(9):2452-7. doi: 10.1519/JSC.0000000000002234.

9. Vaquero-Cristóbal R, Alacid F, Esparza-Ros F, Muyor JM, López-Miñarro PÁ. Características morfológicas y perfil antropométrico en mujeres que practican pilates clásico y mat clásico. Int J Morphol. 2014;32(2):695-702. doi:10.4067/S0717-95022014000200052.

10. Serairi Beji R, Megdiche Ksouri W, Ben Ali R, Saidi O, Ksouri $\mathrm{R}$, Jameleddine S. Evaluation of nutritional status and body composition of young Tunisian weightlifters. Tunis Med. 2016;94(2):112-7. Disponible en: https://pubmed.ncbi.nlm.nih.gov/27532526/

11. Pons V, Riera J, Galilea PA, Drobnic F, Banquells M, Ruiz O. Características antropométricas, composición corporal y somatotipo por deportes. Datos de referencia del CAR de San Cugat, 1989-2013. Apunt Med l'Esport. 2015;50(186):65-72. doi:10.1016/j.apunts.2015.01.002.

12. López C, Aragonés MT. Somatotipo y porcentaje de grasa corporal en halterofilia. Apunts Med Esport. 1989:26(101):151-6. Disponible en: https://www.apunts.org/es-somatotipo-grasa-corporalhalterofilia-articulo-X0213371789050739

13. Fernández Vieitez JA, Ricardo Aguilera R. Estimación de la masa muscular por diferentes ecuaciones antropométricas en levantadores de pesas de alto nivel. Arch med Deport. 2001;18(86):585-91.

Disponible

en: https://pesquisa.bvsalud.org/portal/resource/pt/ibc23214?lang=es

14. Rodríguez PX, Castillo VO, Tejo CJ, Rozowski NJ. Somatotipo de los deportistas de alto rendimiento de Santiago, Chile. Rev Chil Nutr. 2014;41(1):29-39. doi:10.4067/S071775182014000100004.

15. da Silva VS, Vieira MF. International society for the advancement of kinanthropometry (Isak) global: International accreditation scheme of the competent anthropometrist. Rev Bras Cineantropometria e Desempenho Hum. 2020;22:e70517. doi: 10.1590/1980$0037.2020 \mathrm{v} 22 \mathrm{e} 70517$.

16. Ross WD, Kerr DA. Fraccionamiento de la masa corporal: Un nuevo método para utilizar en nutrición clínica y medicina deportiva. Apunts Sports Med. 1991;28(109):175-88. Disponible en: https://www.apunts.org/enfraccionamiento-masa-corporal-un-nuevo-articuloX0213371791052237

17. Bazyler CD, Mizuguchi S, Zourdos MC, Sato K, Kavanaugh AA, DeWeese BH, et al. Characteristics of a national level female weightlifter peaking for competition: a case study. J Strength Cond Res. 2018;32(11):3029-38. doi: 10.1519/JSC.0000000000002379.

18. Ahtiainen JP, Walker S, Peltonen H, Holviala J, Sillanpää E, Karavirta L, et al. Heterogeneity in resistance traininginduced muscle strength and mass responses in men and women of different ages. Age (Omaha). 2016;38(1):1-13. doi: $10.1007 / \mathrm{s} 11357-015-9870-1$.

19. Zamboni M, Turcato E, Santana H, Maggi S, Harris TB, Pietrobelli A, et al. The relationship between body composition and physical performance in older women. J Am Geriatr Soc. 1999;47(12):1403-8. doi:10.1111/j.15325415.1999.tb01557.x.

20. Dao H, Kazin RA. Gender differences in skin: a review of the literature. Gend Med. 2007;4(4):308-28. doi:10.1016/s1550-8579(07)80061-1.

21. Snyder PJ, Kopperdahl DL, Stephens-Shields AJ, Ellenberg SS, Cauley JA, Ensrud KE, et al. Effect of testosterone treatment on volumetric bone density and strength in older men with low testosterone: A controlled clinical trial. JAMA Intern Med. 2017;177(4):471-9. doi:10.1001/jamainternmed.2016.9539.

22. Herrera-Valenzuela $T$, Valdés-Badilla $P$, Soto-Voisier $E$, Verdugo-Miranda F, Cancino-López J, Sáez-Fuentes M, et al. Rapid weight loss: The case of combat sports. Rev. méd Chile. 2018;146(8):947-8. doi: 10.4067/s003498872018000800947. 